\title{
Coincidence points of mappings and relations with applications
}

\author{
Akbar Azam
}

Correspondence:

akbarazam@yahoo.com

Department of Mathematics,

COMSATS Institute of Information

Technology, Chak Shahzad, 44000

Islamabad, Pakistan

\section{Springer}

\begin{abstract}
We obtain coincidence points of mappings and relations under a contractive condition in a metric space. As applications, we achieve an existence and uniqueness theorem of solution for a general class of nonlinear integral equations. 2010 Mathematics Subject Classification: 47H10; 54H25; 54C60.
\end{abstract}

Keywords: coincidence point, contractive mappings, relations, Fredholm type, integral equation

\section{Introduction}

The advancement and the rich growth of fixed point theorems in metric spaces has important theoretical and practical applications. This developments in the last three decades were tremendous. For most of them, their reference result is the Banach contraction theorem, which states that if $X$ is a complete metric space and $T: X \rightarrow X$ a contractions mapping on $X$ (i.e., $d(T x, T y) \leq \lambda d(x, y)$ for all $x, y \in X$, where $0<\lambda<1$ ), then $T$ has a unique fixed point in $X$ (see also [1], Lemma 1]). This theorem looks simple but plays a fundamental role in fixed point theory [2]. Jungck [3] studied coincidence and common fixed points of commuting mappings and improved the Banach contraction principle. The coincidence and common fixed points generalizations were further studied by many authors (e.g., see [4-6]). In addition, see Kirk [7], Murthy [8], Park [9,10], and Rhoades [11,12], for a survey of this subject. Currently Aydi et al. [13] established some coincidence and common fixed point results for three self-mappings on a partially ordered cone metric space satisfying a contractive condition and proved an existence theorem of a common solution of integral equations. In the same way, Shatanawi et al. [14] studied some new real generalizations on coincidence points for weakly decreasing mappings satisfying a weakly contractive condition in an ordered metric space.

On the other hand Haghi et al. [15] showed that some coincidence point and common fixed point generalizations for two mappings in fixed point theory are not real generalizations and they obtained some coincidence and common fixed point results for two self mappings from their corresponding fixed point theorems.

In the present article, we prove the existence of a coincidence point of a mapping and a relation under a contractive condition which is an innovative and real generalization of the Banach contraction theorem. Moreover, a result is deduced on existence of a unique coincidence point for two nonself mappings under a contractive condition.

(C) 2012 Azam; licensee Springer. This is an open access article distributed under the terms of the Creative Commons Attribution License (http://creativecommons.org/licenses/by/2.0), which permits unrestricted use, distribution, and reproduction in any medium, provided the original work is properly cited. 
As applications, we achieve an existence and uniqueness theorem of solution for a class of nonlinear integral equations.

\section{Preliminaries}

Let $A$ and $B$ be arbitrary nonempty sets. A relation $R$ from $A$ to $B$ is a subset of $A \times B$ and is denoted by $R: A \leadsto B$. The statement $(x, y) \in R$ is read " $x$ is $R$-related to $y^{\prime \prime}$, and is denoted by $x R y$. $A$ relation $R: A \leadsto B$ is called left-total if for all $x \in A$ there exists a $y \in B$ such that $x R y . R$ is called right-total if for all $y \in B$ there exists an $x \in$ $A$ such that $x R y . R$ is known as functional, if $x R y, x R z$ implies that $y=z$, for $x \in A$ and $y, z \in B$. A mapping $T: A \rightarrow B$ is a relation from $A$ to $B$ which is both functional and left-total. For $R: A \leadsto B, E \subset A$ we define

$$
\begin{gathered}
R(E)=\{y \in B: x R y \text { for some } x \in E\} . \\
\quad \operatorname{dom}(R)=\{x \in A: R(\{x\}) \neq \phi\},
\end{gathered}
$$

Range $(R)=\{y \in B: y \in R(\{x\})$ for some $x \in \operatorname{dom}(R)\}$.

For convenience, we denote $R(\{x\})$ by $R\{x\}$. The class of relations from $A$ to $B$ is denoted by $\mathcal{R}(A, B)$. Thus the collection $\mathcal{M}(A, B)$ of all mappings from $A$ to $B$ is a proper sub collection of $\mathcal{R}(A, B)$.. An element $w \in A$ is called coincidence point of $T$ : $A \rightarrow B$ and $R: A \rightsquigarrow B$ if $T w \in R\{w\}$. In the following, we always suppose that $X$ is nonempty set and $(Y, d)$ is a metric space. For $R: X \rightsquigarrow Y$ and $u, v \in \operatorname{dom}(R)$, we define

$$
D(R\{u\}, R\{v\})=\inf _{u R x, v R y} d(x, y)
$$

A function $\Psi:[0, \infty) \rightarrow[0,1)$ is said to have property (p) [16-18] if for $t>0$, there exists $\delta(t)>0, s(t)<1$ such that

$$
0 \leq r-t<\delta(t) \Rightarrow \Psi(r) \leq s(t) .
$$

\section{Coincidence points}

Theorem 3.1 Let $X$ be a nonempty set and $(Y, d)$ be a metric space. Let $T: X \rightarrow Y, R$ : $X \leadsto Y$ be such that $R$ is left-total, Range $(T) \subseteq$ Range $(R)$ and Range $(T)$ or Range $(R)$ is complete. If there exists a non-decreasing function $\Psi:[0, \infty) \rightarrow[0,1)$ having property (p) such that for all $x, y \in X$

$$
d(T x, T y) \leq \Psi(D(R\{x\}, R\{y\})) D(R\{x\}, R\{y\}) .
$$

Then there exists $w \in X$ such that $T w \in R\{w\}$.

Proof. Let $x_{0}$ be an arbitrary, but fixed element of $X$. We shall construct sequences $\left\{x_{n}\right\} \subset X,\left\{y_{n}\right\} \subset$ Range $(R)$. Let $y_{1}=T x_{0}$, using the fact that Range $(T) \subseteq$ Range $(R)$, we may choose $x_{1} \in X$ such that

$$
x_{1} R y_{1} \text {. }
$$

Let $y_{2}=T x_{1}$, if

$$
\Psi\left(D\left(R\left\{x_{0}\right\}, R\left\{x_{1}\right\}\right)\right) D\left(R\left\{x_{0}\right\}, R\left\{x_{1}\right\}\right)=0,
$$

then by assumptions $T x_{0}=T x_{1}$. It implies that

$$
x_{1} R y_{2} \text {. }
$$


Then $x_{1}$ is the point of $X$ we are looking for. If

$$
\Psi\left(D\left(R\left\{x_{0}\right\}, R\left\{x_{1}\right\}\right) D\left(R\left\{x_{0}\right\}, R\left\{x_{1}\right\}\right) \neq 0,\right.
$$

then using inequality (1) we have

$$
d\left(T x_{0}, T x_{1}\right) \leq \Psi\left(D\left(R\left\{x_{0}\right\}, R\left\{x_{1}\right\}\right)\right) D\left(R\left\{x_{0}\right\}, R\left\{x_{1}\right\}\right) \neq 0 .
$$

Choose $x_{2} \in X$ such that $x_{2} R y_{2}$. In the case

$$
\Psi\left(D\left(R\left\{x_{1}\right\}, R\left\{x_{2}\right\}\right)\right) D\left(R\left\{x_{1}\right\}, R\left\{x_{2}\right\}\right)=0,
$$

$x_{2}$ is the required point in $X$. If

$$
\Psi\left(D\left(R\left\{x_{1}\right\}, R\left\{x_{2}\right\}\right)\right) D\left(R\left\{x_{1}\right\}, R\left\{x_{2}\right\}\right) \neq 0,
$$

then inequality (1) implies that

$$
d\left(T x_{1}, T x_{2}\right) \leq \Psi\left(D\left(R\left\{x_{1}\right\}, R\left\{x_{2}\right\}\right)\right) D\left(R\left\{x_{1}\right\}, R\left\{x_{2}\right\}\right) \neq 0,
$$

By induction we produce sequences $\left\{x_{n}\right\} \subset X$ and $\left\{y_{n}\right\} \subset$ Range $(R)$ such that $y_{n}=$ $T x_{n-1}, x_{n} R y_{n}$ and

$$
d\left(y_{n}, y_{n+1}\right) \leq \Psi\left(D\left(R\left\{x_{n-1}\right\}, R\left\{x_{n}\right\}\right) D\left(R\left\{x_{n-1}\right\}, R\left\{x_{n}\right\}\right) \neq 0, n=1,2,3, \ldots\right.
$$

Since $x_{n} R y_{n}, x_{n+1} R y_{n+1}$ therefore, by definition of $D$, we have

$$
D\left(R\left\{x_{n}\right\}, R\left\{x_{n+1}\right\}\right) \leq d\left(y_{n}, y_{n+1}\right) \text {. }
$$

Thus,

$$
d\left(y_{n+1}, y_{n+2}\right) \leq \Psi\left(d\left(y_{n}, y_{n+1}\right) d\left(y_{n}, y_{n+1}\right) .\right.
$$

It follows that

$$
d\left(y_{n+1}, y_{n+2}\right)<d\left(y_{n}, y_{n+1}\right), n=1,2,3, \ldots
$$

Thus,

$$
\lim _{n \rightarrow \infty} d\left(y_{n}, y_{n+1}\right)=\inf \left\{d\left(y_{n}, y_{n+1}\right): n \geq 0\right\} .
$$

Assume that

$$
\lim _{n \rightarrow \infty} d\left(y_{n}, y_{n+1}\right)=t
$$

We claim $t=0$. Otherwise by property $(p)$ of $\Psi$, there exists $\delta(t)>0, s(t)<1$, such that

$$
0 \leq r-t<\delta(t) \Rightarrow \Psi(r) \leq s(t)<1
$$

For this $\delta(t)>0$, there exists a natural number $N$ such that

$$
0 \leq d\left(y_{n}, y_{n+1}\right)-t<\delta(t), \text { whenever } n \geq N \text {. }
$$

Hence,

$$
\Psi\left(d\left(y_{n}, y_{n+1}\right)\right) \leq s(t), \text { whenever } n \geq N .
$$


Then inequality (1) implies that

$$
d\left(y_{n}, \gamma_{n+1}\right) \leq \max \left\{\Psi\left(d\left(y_{0}, \gamma_{1}\right)\right), \Psi\left(d\left(y_{1}, \gamma_{2}\right)\right), \ldots, \Psi\left(d\left(y_{N-1}, y_{N}\right)\right), s(t)\right\} d\left(y_{n-1}, y_{n}\right) .
$$

Assume that

$$
M=\max \left\{\Psi\left(d\left(y_{0}, y_{1}\right)\right), \Psi\left(d\left(y_{1}, y_{2}\right), \ldots, \Psi\left(d\left(y_{N-1}, y_{N}\right)\right), s(t)\right\} .\right.
$$

Then $M<1$ and

$$
d\left(y_{n}, y_{n+1}\right) \leq M d\left(y_{n-1}, y_{n}\right) \text { for } n=1,2,3, \ldots
$$

Hence

$$
d\left(y_{n}, y_{n+1}\right) \leq M^{n} d\left(y_{0}, y_{1}\right) \rightarrow 0 \text { as } n \rightarrow \infty,
$$

this contradicts the assumption that $t>0$. Consequently

$$
\lim _{n \rightarrow \infty} d\left(y_{n}, y_{n+1}\right)=0 .
$$

Now we prove that $\left\{y_{n}\right\}$ is a Cauchy sequence. Assume that $\left\{y_{n}\right\}$ is not a Cauchy sequence. Then there exists a positive number $t^{*}$ and subsequences $\{n(i)\},\{m(i)\}$ of the natural numbers with $n(i)<m(i)$ such that

$$
d\left(y_{n(i)}, y_{m(i)}\right) \geq t^{*}, d\left(y_{n(i)}, y_{m(i)-1}\right)<t^{*},
$$

for $i=1,2,3, \ldots$. Then

$$
\begin{aligned}
t^{*} & \leq d\left(y_{n(i)}, y_{m(i)}\right) \\
& \leq d\left(y_{n(i)}, y_{m(i)-1}\right)+d\left(y_{m(i)-1}, y_{m(i)}\right) .
\end{aligned}
$$

Letting $i \rightarrow \infty$ and using the fact that $d\left(y_{n(i)}, y_{m(i)-1}\right)<t^{*}$, we obtain

$$
\lim _{n \rightarrow \infty} d\left(y_{n(i)}, y_{m(i)}\right)=t^{*} \text {. }
$$

For this $t^{*}>0$, by property (p) of $\Psi$ there exists $\delta\left(t^{*}\right)>0, s\left(t^{*}\right)<1$, such that

$$
0 \leq r-t^{*}<\delta\left(t^{*}\right) \Rightarrow \Psi(r) \leq s\left(t^{*}\right)<1 .
$$

For this $\delta\left(t^{*}\right)>0$, there exists a natural number $N_{0}$ such that

$$
0 \leq d\left(y_{n(i)}, y_{m(i)}\right)-t^{*}<\delta\left(t^{*}\right), \text { whenever } i \geq N_{0} .
$$

Hence

$$
i \geq N_{o} \Rightarrow \Psi\left(d\left(y_{n(i)}, y_{m(i)}\right)\right) \leq s\left(t^{*}\right) .
$$

Now, inequality (1) yields

$$
\begin{aligned}
d\left(y_{n(i)}, y_{m(i)}\right) & \leq d\left(y_{n(i)}, y_{n(i)+1}\right)+d\left(y_{n(i)+1}, y_{m(i)+1}\right)+d\left(y_{m(i)+1}, y_{m(i)}\right) \\
& \leq d\left(y_{n(i)}, y_{n(i)+1}\right)+\Psi\left(d\left(y_{n(i)}, y_{m(i)}\right)\right) d\left(y_{n(i)}, y_{m(i)}\right)+d\left(y_{m(i)+1}, y_{m(i)}\right) \\
& \leq d\left(y_{n(i)}, y_{n(i)+1}\right)+\Psi\left(d\left(y_{n(i)}, y_{m(i)}\right)\right) d\left(y_{n(i)}, y_{m(i)}\right)+d\left(y_{m(i)+1}, y_{m(i)}\right) .
\end{aligned}
$$

Thus

$$
d\left(y_{n(i)}, y_{m(i)}\right) \leq d\left(y_{n(i)}, y_{n(i)+1}\right)+s\left(t^{*}\right) d\left(y_{n(i)}, y_{m(i)}\right)+d\left(y_{m(i)+1}, y_{m(i)}\right)
$$


Letting $i \rightarrow \infty$, we get

$$
t^{*} \leq s\left(t^{*}\right) t^{*}<t^{*}
$$

a contradiction. Hence $\left\{y_{n}\right\}$ is a Cauchy sequence in Range $(R)$. By completeness of this space there exists an element $z \in \operatorname{Range}(R)$ such that $y_{n} \rightarrow z$. It further implies that $w R z$ for some $w \in X$. Now,

$$
\begin{aligned}
d(z, T w) & \leq d\left(z, y_{n}\right)+d\left(y_{n}, T w\right) \\
& \leq d\left(z, y_{n}\right)+d\left(T x_{n-1}, T w\right) \\
& \leq d\left(z, y_{n}\right)+\Psi\left(D\left(R\left\{x_{n-1}\right\}\right), R\{w\}\right) D\left(R\left\{x_{n-1}\right\}, R\{w\}\right) \\
& <d\left(z, y_{n}\right)+D\left(R\left\{x_{n-1}\right\}, R\{w\}\right) \\
& <d\left(z, y_{n}\right)+d\left(y_{n-1}, z\right) .
\end{aligned}
$$

Letting $n \rightarrow \infty$, we have $d(z, T w)=0$. It follows that $z=T w$. Hence $T w \in R\{w\}$. In the case when Range $(T)$ is complete. The fact Range $(T) \subseteq$ Range $(R)$ implies that there exists an element $z^{*} \in \operatorname{Range}(R)$ such that $y_{n} \rightarrow z^{*}$. The remaining part of the proof is same as in previous case.

Example 3.2 Let $X=Y=R, d(x, y)=|x-y|$. Define $T: R \rightarrow R, R: R \leadsto R$ as follows:

$$
\begin{gathered}
T x=\left\{\begin{array}{l}
1 \text { if } x \in \mathbb{Q} \\
0 \text { if } x \in \mathbb{Q}^{\prime},
\end{array}\right. \\
R=(\mathbb{Q} \times[0,4]) \cup\left(\mathbb{Q}^{\prime} \times[7,9]\right)
\end{gathered}
$$

Then Range $(T)=\{0,1\} \subset$ Range $(R)=[0,4] \cup[7,9]$. For $\Psi(t)=\frac{1}{3}$, all conditions of the above theorem are satisfied.

From Theorem 3.1, we deduce the following result immediately.

Theorem 3.3 Let $X$ be nonempty set and $(Y, d)$ be a metric space. Let $T: X \rightarrow Y, R$ : $X \leadsto Y$ be such that $R$ is left-total, Range $(T) \subseteq$ Range $(R)$ and Range $(T)$ or Range $(R)$ is complete. If there exists $\lambda \in[0,1)$ such that for all $x, y \in X$

$$
d(T x, T y) \leq \lambda D(R\{x\}, R\{y\}) .
$$

Then there exists $w \in X$ such that $T w \in R\{w\}$.

In the following theorem, we prove the existence of a unique coincidence point of a pair of nonself mappings under a contractive condition.

Theorem 3.4 Let $X$ be a nonempty set and $(Y, d)$ be a metric space. T, $S: X \rightarrow Y$ be two mappings such that Range $(T) \subseteq$ Range $(S)$ and Range $(T)$ or Range $(S)$ is complete. If there exists $a \lambda \in[0,1)$ such that for all $x, y \in X$

$$
d(T x, T y) \leq \lambda d(S x, S y)
$$

Then $S$ and $T$ have a coincidence point in X. Moreover, if either $T$ or $S$ is injective, then $S$ and $T$ have a unique coincidence point in $X$.

Proof. By Theorem 3.1, we obtain that there exists $w \in X$ such that $T w=S w$, where,

$$
S w=\lim _{x \rightarrow \infty} S x_{n}=\lim _{x \rightarrow \infty} T x_{n-1}, x_{0} \in X .
$$

For uniqueness, assume that $w_{1}, w_{2} \in X, w_{1} \neq w_{2}, T w_{1}=S w_{1}$, and $T w_{2}=S w_{2}$. Then $d\left(T w_{1}, T w_{2}\right) \leq \lambda d\left(S w_{1}, S w_{2}\right)$. If $S$ or $T$ is injective, then 


$$
d\left(S w_{1}, S w_{2}\right)>0
$$

and

$$
d\left(S w_{1}, S w_{2}\right)=d\left(T w_{1}, T w_{2}\right) \leq \lambda d\left(S w_{1}, S w_{2}\right),
$$

a contradiction.

Remark 3.5 If in the above theorem we choose $X=Y$, and $S=I$ (the identity mapping on $X$ ), we obtain the Banach contraction theorem.

\section{Integral equations}

The purpose of this section is to study the existence and uniqueness of solution of a general class of Fredholm integral equations of 2 nd kind under various assumptions on the functions involved. Theorem 3.4 coupled with a function space $(C[a, b], \mathbb{R})$ and a contractive inequality are used to establish the result. Consider the integral equation:

$$
f x(t)-\mu \int_{a}^{b} K(t, s) h x(s) d s=g(t)
$$

were, $x:[a, b] \rightarrow \mathbb{R}$ is unknown, $g:[a, b] \rightarrow \mathbb{R}$ and $h, f: \mathbb{R} \rightarrow \mathbb{R}$ are given, $\mu$ is a parameter. The kernel $K$ of the integral equation is defined on $[a, b] \times[a, b]$. If $f=h$ $=I$ (the identity mapping on $\mathbb{R}$ ), then (2) is Known as Fredholm integral equation of 2nd kind (see also [19] and the references cited therein).

Theorem 4.1 Let $K, f, g, h$ be continuous. Let $c\lfloor R$ such that, for all $t, s \in[a, b]$

$$
|K(t, s)| \leq c
$$

and for each $x \in(C[a, b], \mathbb{R})$ there exists $y \in(C[a, b], \mathbb{R})$ such that

$$
(f y)(t)=g(t)+\mu \int_{a}^{b} K(t, s) h x(s) d s .
$$

If $f$ is injective, there exists $L \in R$ such that for all $x, y \in R$

$$
|h x-h y| \leq L|f x-f y|
$$

and $\{f x: x \in(C[a, b], \mathbb{R})\}$ is complete. Then, for $\mu \in\left(-\frac{1}{c(b-a) L}, \frac{1}{c(b-a) L}\right)$, there exists $w \in(C[a, b], \mathbb{R})$ such that for $x_{0} \in(C[a, b], \mathbb{R})$,

$$
f w(t)=\lim _{x \rightarrow \infty} f x_{n}(t)=\lim _{x \rightarrow \infty}\left[g(t)+\mu \int_{a}^{b} K(t, s) h x_{n-1}(s) d s\right],
$$

and $w$ is the unique solution of (2).

Proof. Let $X=Y=(C[a, b], \mathbb{R})$ and $d(x, y)=\max _{t \in[a, b]}|x(t)-\gamma(t)|$ for all $x, y \in X$. Let $T$, $S: X \rightarrow X$ be defined as follows:

$$
(T x)(t)=g(t)+\mu \int_{a}^{b} K(t, s)(h x)(s) d s, \quad S x=f x .
$$


Then by assumptions $S X=\{S x: x \in X\}$ is complete. Let $x^{*} \in T X$, then $x^{*}=T x$ for $x$ $\in X$ and $x^{*}(t)=T x(t)$. By assumptions there exists $y \in X$ such that $T x(t)=f y(t)$, hence $T X \subseteq S X$. Since,

$$
\begin{aligned}
|(T x)(t)-(T y)(t)| & =|\mu|\left|\int_{a}^{b}[K(t, s)(h x)(s)] d s-\int_{a}^{b}[K(t, s)(h y)(s)] d s\right| \\
& \leq|\mu| \int_{b}^{b} c|(h x)(s)-(h y)(s)| d s \\
& \leq L|\mu| c \int_{b}^{b}|(f x)(s)-(f y)(s)| d s \\
& \leq L|\mu| c \int_{b}^{b}|(S x)(s)-(S y)(s)| d s \\
& \leq\left(\sup _{t \in[a, b]}|(S x)(t)-(S y)(t)|\right) L|\mu| c\left|\int_{a}^{b} d s\right| \\
& \leq L|\mu| c(b-a) d(S x, S y) .
\end{aligned}
$$

Therefore, for any $\mu \in\left(-\frac{1}{c(b-a) L}, \frac{1}{c(b-a) L}\right)$, all conditions of Theorem 3.4 are satisfied. Hence, there exists a unique $w \in X$ such that

$$
f w(t)=\lim _{x \rightarrow \infty} S x_{n}(t)=\lim _{x \rightarrow \infty} T x_{n-1}(t)=T(w)(t), x_{0} \in X
$$

for all $t$, which is the unique solution of (2).

Example 4.2 Consider the integral equation:

$$
\begin{gathered}
{[3 x(t)]^{3}=\sin t+\mu \int_{0}^{\frac{\pi}{2}}[\sqrt{t} x(s)]^{3} d s} \\
\text { Let } X=Y=\left(C\left[0, \frac{\pi}{2}\right], \mathbb{R}\right), d(x, y)=\max _{t \in C\left[0, \frac{\pi}{2}\right]}|x(t)-y(t)|_{\text {for all } x, y \in X . \text { Since, }} \\
|K(t, s)|=\left|t^{\frac{3}{2}} s^{0}\right| \leq\left(\frac{\pi}{2}\right)^{\frac{3}{2}}
\end{gathered}
$$

and

$$
\left|x^{3}(t)-y^{3}(t)\right| \leq \frac{1}{27}\left|[3 x(t)]^{3}-[3 y(t)]^{3}\right|
$$

for all $x, y \in R$, therefore all conditions of Theorem 4.2 are satisfied for $c=\left(\frac{\pi}{2}\right)^{\frac{3}{2}}, h(x)=x^{3}, f(x)=27 x^{3}, g(x)=\sin x, L=\frac{1}{27}$ Hence for $\mu \in\left(-\frac{27}{\left(\frac{\pi}{2}\right)^{\frac{5}{2}}}, \frac{27}{\left(\frac{\pi}{2}\right)^{\frac{5}{2}}}\right)$, there exists a unique solution of (4). We approximate the solution, by constructing the iterative sequences: 


$$
S x_{n}=T x_{n-1}, x_{0} \in X, n=1,2,3 \ldots
$$

in connection with the mappings $S, T: X \rightarrow X$ defined as follows:

$$
(T x)(t)=\sin t+\mu \int_{0}^{\frac{\pi}{2}}[\sqrt{t} x(s)]^{3} d s, \quad(S x)(t)=27 x^{3}(t) .
$$

Let $x_{0}:\left[0, \frac{\pi}{2}\right] \rightarrow \mathbb{R}$ be defined as $x_{0}(t)=0$. Then

$$
\left(T x_{0}\right)(t)=\sin t=\left(S x_{1}\right)(t) .
$$

It follows that

$$
x_{1}(t)=\frac{1}{3}(\sin t)^{\frac{1}{3}} .
$$

Now

$$
\begin{aligned}
\left(T x_{1}\right)(t) & =\sin t+\mu \int_{0}^{\frac{\pi}{2}}\left[\sqrt{t} \frac{1}{3}(\sin s)^{\frac{1}{3}}\right]^{3} d s \\
& =\sin t+\mu \frac{1}{27} \int_{0}^{\frac{\pi}{2}} t^{\frac{3}{2}} \sin s d s \\
& =\sin t+\frac{1}{27} \mu t^{\frac{3}{2}}=\left(S x_{2}\right)(t) .
\end{aligned}
$$

It implies that

$$
x_{2}(t)=\frac{1}{3}\left[\sin t+\frac{1}{27} \mu t^{\frac{3}{2}}\right]^{\frac{1}{3}} .
$$

Similarly,

$$
\left(T x_{n}\right)(t)=\sin t+\sum_{j=1}^{n} t^{\frac{3}{2}} \frac{1}{27^{j}} \mu^{j}\left(\frac{2}{5}\right)^{(j-1)}\left(\frac{\pi}{2}\right)^{(j-1) \frac{5}{2}}=\left(S x_{n+1}\right)(t) .
$$

As $\mu \in\left(-\frac{27}{\left(\frac{\pi}{2}\right)^{\frac{5}{2}}}, \frac{27}{\left(\frac{\pi}{2}\right)^{\frac{5}{2}}}\right)$, the series $\sum_{j=1}^{\infty} t^{\frac{3}{2}} \frac{1}{27^{j}} \mu^{j}\left(\frac{2}{5}\right)^{(j-1)}\left(\frac{\pi}{2}\right)^{(j-1) \frac{5}{2}}$ is convergent and

$$
\lim _{x \rightarrow \infty} S x_{n}(t)=\sin t+\left(\frac{\frac{1}{27} \mu t^{\frac{3}{2}}}{1-\frac{1}{27} \mu \frac{2}{5}\left(\frac{\pi}{2}\right)^{\frac{5}{2}}}\right)=S w(t) .
$$

Hence,

$$
\frac{1}{3}\left[\sin t+\left(\frac{\frac{1}{27} \mu t^{\frac{3}{2}}}{1-\frac{1.2}{27.5}\left(\frac{\pi}{2}\right)^{\frac{5}{2}}}\right)\right]^{\frac{1}{3}}=w(t)
$$

is the required solution. 
Acknowledgements

The author was thankful to the editor and the referees for their useful comments and suggestions.

\section{Competing interests}

The author declares that he have no competing interests.

Received: 14 October 2011 Accepted: 26 March 2012 Published: 26 March 2012

\section{References}

1. Liu, W, Fu, C, Hu, H: Global exponential stability of a class of Hopfield neural network with delays. Neural Comput \& Appl. doi:10.1007/s00521-010-0470-9

2. Goebel, K, Kirk, WA: Topics in Metric Fixed Point Theory. Cambridge University Press, Cambridge (1990)

3. Jungck, G: Commuting mappings and fixed points. Amer Math Monthly. 83, 261-263 (1976). doi:10.2307/2318216

4. Beg, I, Azam, A: Fixed points of asymptotically regular multivalued mappings. J Austral Math Soc (ser. A). 53, 313-326 (1992). doi:10.1017/S1446788700036491

5. Daffer, PZ, Kaneko, H: Multivalued f-contractive mappings. Boll Un Mat Ital. 8-A, 233-241 (1994)

6. Pant, RP: Common fixed points for contractive maps. J Math Anal Appl. 226, 281-286 (1998)

7. Kirk, WA: Fixed point theory for non-expansive mappings II. Contemporary Math. 18, 121-140 (1983)

8. Murthy, P: Important tools and possible applications of metric fixed point theory. Nonlinear Anal. 47, 3479-3490 (2001). doi:10.1016/50362-546X(01)00465-5

9. Park, S: Ninety years of the Brouwer fixed point theorem. Vietnam J Math. 27, 193-232 (1999)

10. Park, S: Recent results in analytical fixed point theory. Nonlinear Anal. 63, 977-986 (2005). doi:10.1016/j.na.2005.02.026

11. Rhoades, BE: A comparison of various definitions of contractive mappings. Trans Am Math Soc. 26, 257-290 (1977)

12. Rhoades, BE: Contractive definitions and continuity. Contemporary Math. 12, 233-245 (1988)

13. Aydi, H, Nashine, HK, Samet, B, Yazidi, H: Coincidence and common fixed point results in partially ordered cone metric spaces and applications to integral equations. Nonlinear Anal. 74, 6814-6825 (2011). doi:10.1016/..na.2011.07.006

14. Shatanawi, W, Mustafa, Z, Tahat, N: Some coincidence point theorems for nonlinear, contraction in ordered metric spaces. Fixed Point Theory and Applications 2011, 68 (2011). doi:10.1186/1687-1812-2011-68. doi:10.1186/1687-18122011-68

15. Haghi, RH, Rezapour, Sh, Shahzad, N: Some fixed point generalizations are not real generalizations. Nonlinear Anal. 74, 1799-1803 (2011). doi:10.1016/j.na.2010.10.052

16. Azam, A, Arshad, M: Fixed points of sequence of locally contractive multivalued maps. Comp Math Appl. 57, 96-100 (2008)

17. Azam, A, Beg, I: Common fixed points of fuzzy maps. Math Comp Model. 49, 1331-1336 (2009). doi:10.1016/j. $\mathrm{mcm} .2008 .11 .011$

18. Hu, T: Fixed point theorems for multivalued mappings. Canad Math Bull. 23, 193-197 (1980). doi:10.4153/CMB-1980026-2

19. Corduneanu, C: Integral Equations and Applications. Cambridge University Press, Cambridge (1991)

doi:10.1186/1687-1812-2012-50

Cite this article as: Azam: Coincidence points of mappings and relations with applications. Fixed Point Theory and Applications 2012 2012:50

\section{Submit your manuscript to a SpringerOpen ${ }^{\circ}$ journal and benefit from:}

- Convenient online submission

Rigorous peer review

- Immediate publication on acceptance

- Open access: articles freely available online

- High visibility within the field

- Retaining the copyright to your article

Submit your next manuscript at $\boldsymbol{s p r i n g e r o p e n . c o m ~}$ 